\title{
About the Moving Force of Chemical Reactions
}

\author{
Utelbayev BT ${ }^{1}$, Suleimenov $\mathrm{EN}^{1}$ and Utelbayeva $\mathrm{AB}^{2 *}$ \\ ${ }^{1}$ Kazakhstan-British Technical University, Kazakhstan \\ ${ }^{2}$ M Auezov South Kazakhstan State University, Kazakhstan \\ *Corresponding author: Utelbayeva AB, M Auezov South Kazakhstan State University, Kazakhstan
}

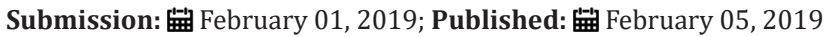

\section{Opinion}

Chemistry is well known-the science of substances that studies the processes of their transformation accompanied by changes in the composition and structure [1-3]. As a result of a chemical reaction, chemical work is performed with the redistribution of chemical bonds, accompanied by the release or absorption of heat, light, and other manifestations. And here the question arises about the nature of the moving force of the work, what is, the reasons for the occurrence of a chemical reaction. At the same time, the number of nucleons, electrons participating in chemical transformations remains unchanged, however, new "chemical individuals" are formed [4-11] and the internal energy of the reacting systems changes. In [2], the authors of the theory of elementary interactions, the course of chemical reactions, conclude that molecules break down into active particles that interact with the molecule in three stages: association, electronic isomerization and dissociation. At their suggestion, the presence of the electron isomerization stage explains why and how the chemical bond-breaking reactions proceed at room temperature. And temperature, catalyst and other factors, with all their physical differences, play the same rolethe initiator. In our opinion, in the case under consideration, the association of reacting molecules is a modification of the theory of the transition state and most importantly, the moving force of electron isomerization is not indicated. It can react only when an active particle appears, for which radicals, ions and solvent molecules are taken, i.e., it also requires activation energy.

In this regard, the elementary carriers of heat, light, etc. we propose-"electromagnetic particles" [12-16] provide the basis for revising the structure of atomic-molecular structures and expanding the range of information about the phenomena occurring. It is also noted here that the effect on physicochemical systems by elemental energy carriers leads to a change in the chemical potential of substances, which is reflected in their micro-macroscopic properties. At the same time, in the structure of "chemical individuals" of a substance, due to the movement of electrons and elementary particles, polarization occurs and the appearance of a potential difference. Similar phenomena occur in photosystems as well in biosystems [17]. In our opinion, these polarized areas of reacting systems form micro-galvanic pairs and are the moving force of chemical interaction. In turn, when exposed to the system with energy, the chemical potential of the system passes into another state, expressed by "thermochemical potential", which can be expressed Gibbs's fundamental equations for open systems with the constancy of the corresponding parameters:

where $\mu \mathrm{i}$ is the "thermochemical potential" of the i-th kind of "chemical individuals"; $\partial \mathrm{ni}$ is an infinitely small change in the number of the i-th type of energy carrier. Usually, the sum of the chemical and electric potentials $(\mu+\mathrm{zF} \varphi)$ is called the electrochemical potential, then the potential that arises on the "chemical individual" during electron movements is called the "thermo electrochemical potential":

$$
\mu \mathrm{i}=\mu+\mathrm{zF} \varphi
$$

where $\mu \mathrm{i}$ and $\mu$ are "thermo-electrochemical" and chemical potentials of a mole of the i-th type of "chemical individuals", J/mol; $\mathrm{z}$ is the number of electrons participating in the elementary act; $\mathrm{F}$ is the Faraday's number, $96500 \mathrm{C} / \mathrm{mol} ; \varphi$-potential difference, $\mathrm{V}$.

To determine the value of the "thermo-electrochemical potential" from the pulsation frequency of the "electromagnetic particle", considering temperature and pressure as intensive quantities, we use the chemical potential equation in the form:

$$
\mu=\mu 0+\mathrm{kT} \ln \mathrm{p}
$$

where $\mu 0$ is the standard chemical potential; $\mathrm{k}$ is the Boltzmann's constant; $\mathrm{T}$ is the thermodynamic temperature; $\mathrm{p}$ is the partial pressure of the pulsating "electromagnetic particle". For a pulsating "electromagnetic particle" particle, the Clapeyron's equation is written in the form:

$$
\mathrm{pVm}=\mathrm{k} \cdot 0.959 \cdot 10^{-11} \cdot \mathrm{v}
$$

where $\mathrm{Vm}$ is the volume of one electromagnet particle creating pressure $\mathrm{p}$; k is the Boltzmann's constant; 0.959-10-11 -temperature coefficient; $v$-oscillation frequency of the "electromagnetic particle". Analysis of the equation allows us to determine the physical meaning of the "p" for an elementary particle, which is 
the "elastic force - p" necessary for the pulsation of an elementary particle in its volume $\mathrm{Vm}, \mathrm{T}$ is the thermodynamic temperature of the system, which is an indicator of thermal equilibria with the environment. The change of $\mathrm{Vm}$ and $\mathrm{p}$ under compression causes an opposing force according to Newton's third law. Based on the above equations, we can conclude that the change in chemical potential depends mainly on the potential difference and the energy of electromagnetic particles according to the equation:

$$
\mu \mathrm{i}-\mu 0=\mathrm{zF} \varphi+\mathrm{hv} \ln \mathrm{p}
$$

Consequently, the potential difference caused by the polarizing action of the "electromagnetic particle" is the moving force behind the process.

\section{References}

1. (2013) General Chemistry. Wikibooks org.

2. Yu GV (2001) General chemistry. Electronic collection. Russian.

3. https://open.umn.edu/opentextbooks/textbooks/general-chemistryprinciples-patterns-and-applications

4. https://www.gumer.info/bibliotek_Buks/Science/bond/07.php

5. Sirotkin OS, Sirotkin RO (2018) On the evolution of basic chemica concepts chemical individual and molecule in the framework of the development of the Unified Theory of the structure of chemical compounds. Bulletin of the technological university 21(4).

6. Utelbayev B, Suleimenov E, Utelbayeva A, Zhanabay N (2014) Some concepts about substance, chemical compound and an element. American Chemical Science Journal 4(2): 166-173.

7. Sirotkin OS, Sirotkin RO (2001) Higher Education in Russia 6: 137-139.
8. Sirotkin OS (2003) Principles of unified chemistry (Unitarity as the basis for the formation of individuality, the disclosure of the uniqueness and fundamental nature of chemical science). Fan, Kazan, Russia, p. 252.

9. Sirotkin OS (2013) Evolution of the theory of the structure of a chemical substance. In: Butlerov AM (Eds.), in the unitary theory of the structure of chemical compounds. INFRA-M, Moscow, Russia, p. 272.

10. Sirotkin OS, Sirotkin RO (2014) Chemistry (Fundamentals of Unified Chemistry). Knorus, Moscow, Russia, p. 264

11. Ugai YA (1997) General and inorganic chemistry. Higher school, Moscow, Russia, p. 527.

12. Utelbayev BT, Suleimenov EN, Utelbayeva AB (2015) Interconnection of heat and mass changes of the reacting substances at physical and chemical transformations. J Chem Bio and Phy Sci 5(2): 1783-1790.

13. Utelbayev BT, Suleimenov EN, Utelbaeva AB (2016) The nature and mass of elementary particles of heat carriers. International Journal of Scientific Research in Science and Technology 5(6): 6-9.

14. Utelbayev BT, Suleimenov EN, Utelbaeva AB (2016) Temperature is the parameter of the structural and energy state of the system. International Journal of Scientific Latest Research in Science and Technology 5(6): 14-18.

15. Utelbayev BT, Suleimenov EN, Utelbayeva AB (2017) The essence of temperature and its relationship with thermal state of the system. International Journal of Scientific Research in Science and Technology 3(2): 678-684.

16. Utelbayev BT, Suleimenov EN, Utelbaeva AB (2018) The role of elementary particles and the physicochemical properties of material objects. Materials of the international scientific-practical conference. Vologda, Russia.

17. Mitchell P (1961) Coupling of phosphorylation to electron and hydrogen transfer. Nature 191(4784): 144-148.
Creative Commons Attribution 4.0

International License

For possible submissions Click Here

\section{Submit Article}

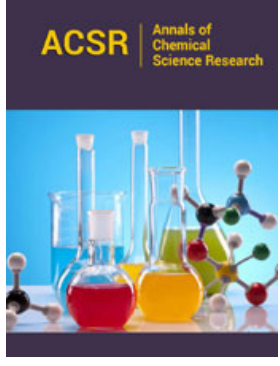

\section{Annals of Chemical Science Research}

\section{Benefits of Publishing with us}

- High-level peer review and editorial services

- Freely accessible online immediately upon publication

- Authors retain the copyright to their work

- Licensing it under a Creative Commons license

- Visibility through different online platforms 